\title{
Instantaneous Characteristics of Nonlinear Torsion Pendulum and Its Application in Parameter Estimation of Nonlinear System
}

\author{
Yan Zhao $\mathbb{D}^{1},{ }^{1}$ Baofeng Zhang, ${ }^{1}$ Fangfang Han, ${ }^{1}$ Huan Tian, $^{2}$ Xiao Yu, ${ }^{1}$ and Junchao Zhu $\mathbb{D}^{1}$ \\ ${ }^{1}$ Tianjin Key Laboratory for Control Theory and Applications in Complicated Systems, Tianjin University of Technology, \\ Tianjin 300384, China \\ ${ }^{2}$ PetroChina Dagang Oilfield Company, Tianjin 300270, China
}

Correspondence should be addressed to Junchao Zhu; zhujunchao_tjut@163.com

Received 21 October 2017; Revised 25 February 2018; Accepted 27 March 2018; Published 10 May 2018

Academic Editor: Suzanne M. Shontz

Copyright @ 2018 Yan Zhao et al. This is an open access article distributed under the Creative Commons Attribution License, which permits unrestricted use, distribution, and reproduction in any medium, provided the original work is properly cited.

\begin{abstract}
The nonlinear model of torsion pendulum is presented by considering the nonlinear damping force and nonlinear restoring force. The analytic solution of the nonlinear model is calculated to analyze the relationship between the characteristics of torsion pendulum and the nonlinear factors. The instantaneous characteristics of nonlinear torsion pendulum are analyzed by instantaneous undamped natural frequency and instantaneous damping coefficient. The instantaneous characteristics can be used for the parameter estimation of nonlinear torsion pendulum system. The nonlinear characteristics of the torsion pendulum are validated by the torsion pendulum based on the air-hovered turntable. The parameter estimation method based on the instantaneous characteristics is validated by the moment of inertia measurement system based on the torsion pendulum.
\end{abstract}

\section{Introduction}

The torsion pendulum system has been widely used to measure the moment of inertia of mechanical systems such as missile and engine [1-4].

The torsion pendulum model mainly contains three factors: moment of inertia, damping force, and restoring force. With the increasing complexity of the torsion pendulum, the linear model has not been able to accurately describe the actual torsion pendulum. For the large measured object with complex shape, the air damping is a nonlinear force related to linear terms and square terms of speed [5]. When the torsion bar is alloy or composite material, the restoring force of the torsion bar is a nonlinear force related to linear terms and cubic terms of torsional angle [6].

The linear model cannot accurately describe the complex torsion pendulum system. So it is needed to establish the torsion pendulum model based on the nonlinear dynamic system. Because the nonlinear system and the linear system have significantly different characteristics, it is necessary to analyze the influence of nonlinear factors on the characteristics of the torsion pendulum.

For the quantitative analysis of the nonlinear torsion pendulum, the approximate analytic solution of the nonlinear differential equation of the torsion pendulum is calculated. The solving methods of the nonlinear differential equation mainly include small parameter method, multiple scales method, evolutionary method, and average method [7-10]. The average method regards the amplitude and phase of the solution as slowly changing with time and uses the derivative of amplitude and phase to compute the approximate analytic solution of the nonlinear differential equation. This method is of high computational accuracy and is suitable for solving nonlinear dynamical model.

The logarithmic decrement and vibration period are the important parameters of the torsion pendulum. The logarithmic decrement refers to the ratio of the amplitude of adjacent periods. For the linear torsion pendulum, the logarithmic decrement and vibration period are constant. But for the nonlinear torsion pendulum, the logarithmic 


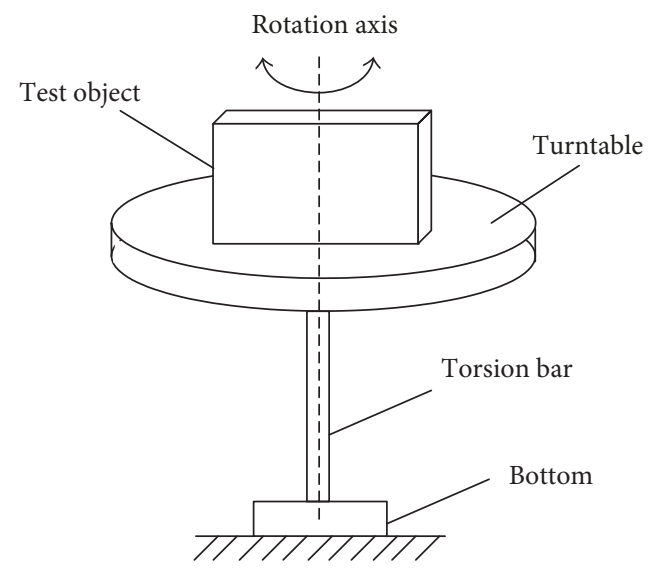

Figure 1: Schematic diagram of torsion pendulum.

decrement and vibration period are time-varying which are associated with nonlinear factors.

The instantaneous characteristics of the nonlinear torsion pendulum can be described by instantaneous undamped natural frequency and instantaneous damping coefficient which are computed by the instantaneous amplitude and instantaneous frequency $[11,12]$.

To calculate the instantaneous frequency, many methods are developed such as short-time Fourier transformation [13, $14]$, wavelet transformation $[15,16]$, Wigner-Vile Distribution $[17,18]$, and Hilbert transform [19]. Reference [11] presented the construction method of analytic signal based on Hilbert transform, which is used for the calculation of instantaneous frequency.

Considering the influence of nonlinear damping force and nonlinear restoring force, the torsion pendulum model based on the nonlinear dynamic system is established. The analytic solution of the nonlinear model is used to analyze the relationship between the characteristics of torsion pendulum and the nonlinear factors. The instantaneous characteristics of the nonlinear system is analyzed by the instantaneous undamped natural frequency and instantaneous damping coefficient. At last, the nonlinear torsion pendulum experimental system is used to verify the presented model and method.

\section{Nonlinear Model of Torsion Pendulum}

The modeling methods based on the differential equation include Newton second law, Darren Bell principle method, and Lagrange equation method. Among them, the Lagrange equation method is based on the energy principle and it only needs to analyze the main power of the system. Therefore, the modeling method based on the Lagrange equation is more suitable for the complex system.

As shown in Figure 1, the torsion pendulum system consists of torsion bar, turntable, and test object. The test object is placed on the turntable that is connected to the torsion bar. With impulse excitation, the test object and turntable make torsion pendulum movement together while the restoring force is generated by the torsion bar. The torsion pendulum system in Figure 1 has one freedom degree and the torsion angle $\theta$ is regarded as the generalized coordinate.

The second Lagrange equation can be expressed as

$$
\frac{d}{d t}\left(\frac{\partial L}{\partial \dot{\theta}}\right)-\frac{\partial L}{\partial \theta}=Q
$$

where $L$ is the Lagrange function, $L=T-V$; $T$ is the kinetic energy of the system; $V$ is the potential energy of the system; $Q$ is the generalized force of the system; and $t$ is the time.

The kinetic energy of the system is

$$
T=\frac{1}{2} J \omega^{2}=\frac{1}{2} J \dot{\theta}^{2}
$$

where $J$ is the moment of inertia with respect to the rotation axis; $\omega$ is the angular speed of the torsion pendulum; $\dot{\theta}$ is the derivative of the torsion angle $\theta$.

The potential energy of the system is

$$
V=\int_{0}^{\theta} K_{1} \theta+K_{3} \theta^{3} d \theta=\frac{1}{2} K_{1} \theta^{2}+\frac{1}{4} K_{3} \theta^{4},
$$

where $K_{1}$ is the linear stiffness coefficient of the torsion bar; $K_{3}$ is the nonlinear stiffness coefficient of the torsion bar; when $K_{3} \neq 0$, the restoring force of the torsion bar is a nonlinear force.

The generalized force of the system is

$$
Q=-C_{1} \dot{\theta}-C_{2} \dot{\theta}|\dot{\theta}|
$$

where $C_{1}$ is the linear damping coefficient; $C_{2}$ is the nonlinear damping coefficient.

Substituting (2)-(4) into (1), we can get the nonlinear model of the torsion pendulum:

$$
J \ddot{\theta}+C_{1} \dot{\theta}+C_{2} \dot{\theta}|\dot{\theta}|+K_{1} \theta+K_{3} \theta^{3}=0 .
$$

Each side of (5) is divided by $J$; the normalization model is expressed as

$$
\ddot{\theta}+c_{1} \dot{\theta}+c_{2} \dot{\theta}|\dot{\theta}|+k_{1} \theta+k_{3} \theta^{3}=0,
$$

where $c_{1}=C_{1} / J ; c_{2}=C_{2} / J ; k_{1}=K_{1} / J$; and $k_{3}=K_{3} / J$.

\section{Analytic Solution of Nonlinear Torsion Pendulum Model}

In order to analyze the characteristics of the nonlinear torsion pendulum, the analytic solution of (5) is calculated by the average method. If the influence of nonlinear factors is weak, a small parameter will appear in the differential equation and the differential equation is typically expressed as

$$
\ddot{\theta}+\omega_{0}^{2} \theta=\varepsilon f(\theta, \dot{\theta}),
$$

where $\varepsilon(\varepsilon \ll 1)$ is a small parameter; $f(\theta, \dot{\theta})$ is the nonlinear function of $\theta$.

Scholar Rylov and Bogoliubov wrote the solution as [20]

$$
\theta=a(t) \cos \left[\omega_{0} t+\varphi(t)\right] .
$$


According to (8), the amplitude $a(t)$ and phase $\varphi(t)$ are regarded as the functions of time. If the influence of the nonlinear factors is weak, the solution can be set as simple harmonic form. as

The first derivative of (8) with respect to time is expressed

$$
\begin{aligned}
\dot{\theta}= & \dot{a}(t) \cos \left[\omega_{0} t+\varphi(t)\right]-a(t) \omega_{0} \sin \left[\omega_{0} t+\varphi(t)\right] \\
& -a(t) \dot{\varphi}(t) \sin \left[\omega_{0} t+\varphi(t)\right] .
\end{aligned}
$$

Because $a(t)$ and $\varphi(t)$ are the slow-variant functions of time, we can get the following equation:

$$
\begin{aligned}
& \dot{a}(t) \cos \left[\omega_{0} t+\varphi(t)\right]-a(t) \dot{\varphi}(t) \sin \left[\omega_{0} t+\varphi(t)\right] \\
& \quad=0 .
\end{aligned}
$$

The first derivative of $\theta(t)$ is expressed as

$$
\dot{\theta}=-a(t) \omega_{0} \sin \left[\omega_{0} t+\varphi(t)\right] .
$$

The second derivative of $\theta(t)$ is expressed as

$$
\begin{aligned}
\ddot{\theta}= & -a(t) \omega_{0}^{2} \cos \left[\omega_{0} t+\varphi(t)\right] \\
& -\dot{a}(t) \omega_{0} \sin \left[\omega_{0} t+\varphi(t)\right] \\
& -a(t) \omega_{0} \dot{\varphi}(t) \cos \left[\omega_{0} t+\varphi(t)\right] .
\end{aligned}
$$

Substituting (8), (11), and (12) into (7) to obtain

$$
\begin{gathered}
-\dot{a}(t) \omega_{0} \sin \psi-a(t) \omega_{0} \dot{\varphi}(t) \cos \psi \\
=\varepsilon f\left(a \cos \psi,-a \omega_{0} \sin \psi\right),
\end{gathered}
$$

where $\psi=\omega_{0} t+\varphi(t)$.

According to (10) and (13), the first-order equation system is expressed as

$$
\begin{aligned}
& \dot{a}(t)=-\frac{\varepsilon}{\omega_{0}} f\left(a \cos \psi,-a \omega_{0} \sin \psi\right) \sin \psi \\
& \dot{\varphi}(t)=-\frac{\varepsilon}{a(t) \omega_{0}} f\left(a \cos \psi,-a \omega_{0} \sin \psi\right) \cos \psi
\end{aligned}
$$

Because $a(t)$ and $\varphi(t)$ are the slow-variant functions of time, the right side of (14) is the periodic function of $\psi$ and they can be expanded as Fourier series. The first term of Fourier series is taken as the approximate value of $\dot{a}(t)$ and $\dot{\varphi}(t):$

$$
\begin{aligned}
& \dot{a}(t) \approx-\frac{\varepsilon}{2 \pi \omega_{0}} \int_{0}^{2 \pi} f\left(a \cos \psi,-a \omega_{0} \sin \psi\right) \sin \psi d \psi \\
& \dot{\varphi}(t) \approx-\frac{\varepsilon}{2 \pi a \omega_{0}} \int_{0}^{2 \pi} f\left(a \cos \psi,-a \omega_{0} \sin \psi\right) \cos \psi d \psi .
\end{aligned}
$$

If the initial condition is given, the value of $a(t)$ and $\varphi(t)$ can be obtained by the integration of (15) and (16).

If (5) is expressed as the form of (7), then

$$
\begin{aligned}
\omega_{0}^{2} & =\frac{K_{1}}{J} \\
\varepsilon f(\theta, \dot{\theta}) & =-\frac{C_{1}}{J} \dot{\theta}-\frac{C_{2}}{J} \dot{\theta}|\dot{\theta}|-\frac{K_{3}}{J} \theta^{3} .
\end{aligned}
$$

Substitute (18) into (15) to obtain

$$
\begin{aligned}
\dot{a}(t) & =-\frac{\varepsilon}{2 \pi \omega_{0}} \int_{0}^{2 \pi} f\left(a \cos \psi,-a \omega_{0} \sin \psi\right) \sin \psi d \psi \\
& =-\frac{\varepsilon}{2 \pi J}\left(C_{1} a \pi+\frac{8}{3} C_{2} a^{2} \omega_{0}\right) .
\end{aligned}
$$

Substituting (18) into (16) to obtain

$$
\begin{aligned}
\dot{\varphi}(t) & =-\frac{\varepsilon}{2 \pi a \omega_{0}} \int_{0}^{2 \pi} f\left(a \cos \psi,-a \omega_{0} \sin \psi\right) \cos \psi d \psi \\
& =-\frac{\varepsilon 3 K_{3} a^{2}}{8 \omega_{0} J} .
\end{aligned}
$$

When $t=0$, the initial value of $a(t)$ is $a_{0}$. By the integration of (19), we can obtain

$$
a(t)=\frac{3 a_{0} \pi C_{1} e^{-\left(\varepsilon C_{1} / 2 J\right) t}}{8 a_{0} C_{2} \omega_{0}+3 \pi C_{1}-8 a_{0} C_{2} \omega_{0} e^{-\left(\varepsilon C_{1} / 2 J\right) t}} .
$$

When $t=0$, the initial value of $\varphi(t)$ is $\varphi_{0}$. By the integration of (20), we can obtain

$$
\varphi(t)=-\frac{\varepsilon 3 K_{3} a^{2}}{8 \omega_{0} J} t+\varphi_{0} .
$$

Substitute (21) and (22) into (8) to obtain the angular displacement $\theta(t)$ :

$$
\begin{aligned}
\theta(t) & =a(t) \cos \left[\omega_{0} t+\varphi(t)\right] \\
= & \left(\frac{3 a_{0} \pi C_{1} e^{-\left(\varepsilon C_{1} / 2 J\right) t}}{8 a_{0} C_{2} \omega_{0}+3 \pi C_{1}-8 a_{0} C_{2} \omega_{0} e^{-\left(\varepsilon C_{1} / 2 J\right) t}}\right) \\
& \cdot \cos \left[\left(\omega_{0}-\frac{\varepsilon 3 K_{3} a^{2}(t)}{8 \sqrt{J K_{1}}}\right) t+\varphi_{0}\right] .
\end{aligned}
$$

According to (21), it is known that the amplitude of the torsion pendulum is related to the damping coefficients $C_{1}$ and $C_{2}$. According to (22), the period of the torsion pendulum is related to the stiffness coefficients of the torsion bars $K_{1}$ and $K_{3}$.

\section{Instantaneous Characteristics of Torsion Pendulum}

4.1. Construction Method of Analytic Signal. If the angular displacement signal of the torsion pendulum is $\theta(t)$, its Hilbert transform $\widetilde{\theta}(t)$ is defined as follows:

$$
\tilde{\theta}(t)=H[\theta(t)]=\frac{1}{\pi} \int_{-\infty}^{+\infty} \frac{\theta(\tau)}{t-\tau} d \tau .
$$

The angular displacement signal $\theta(t)$ and its Hilbert transform $\widetilde{\theta}(t)$ can form the analytic signal $\boldsymbol{\beta}(t)$ :

$$
\boldsymbol{\beta}(t)=\theta(t)+j \tilde{\theta}(t)=A(t) e^{j \Phi(t)},
$$


where instantaneous phase

$$
\Phi(t)=\arctan \left(\frac{\tilde{\theta}(t)}{\theta(t)}\right) .
$$

instantaneous angular frequency

$$
\omega(t)=\frac{d \Phi}{d t},
$$

and instantaneous envelope

$$
A(t)=\sqrt{\theta^{2}(t)+\widetilde{\theta}^{2}(t)} .
$$

The first derivative of analytic signal $\boldsymbol{\beta}(t)$ is as follows:

$$
\dot{\boldsymbol{\beta}}=\boldsymbol{\beta}\left[\frac{\dot{A}(t)}{A(t)}+j \omega(t)\right] .
$$

The second derivative of analytic signal $\boldsymbol{\beta}(t)$ is as follows:

$$
\ddot{\boldsymbol{\beta}}=\boldsymbol{\beta}\left[\frac{\ddot{A}}{A}-\omega^{2}+2 j \frac{\dot{A} \omega}{A}+\mathrm{j} \dot{\omega}\right] .
$$

The calculation formula of the instantaneous angular frequency is as follows:

$$
\omega(t)=\operatorname{Im}\left[\frac{\dot{\boldsymbol{\beta}}(t)}{\boldsymbol{\beta}(t)}\right]=\frac{\theta(t) \dot{\tilde{\theta}}(t)-\dot{\theta}(t) \tilde{\theta}(t)}{\theta^{2}(t)+\widetilde{\theta}^{2}(t)} .
$$

The calculation formula of the first derivative of instantaneous envelope is as follows:

$$
\dot{A}(t)=A(t) \operatorname{Re}\left[\frac{\dot{\boldsymbol{\beta}}(t)}{\boldsymbol{\beta}(t)}\right]=\frac{\theta(t) \dot{\theta}(t)+\widetilde{\theta}(t) \dot{\tilde{\theta}}(t)}{\sqrt{\theta^{2}(t)+\widetilde{\theta}^{2}(t)}} .
$$

4.2. Analysis Method Based on Hilbert Transform. The nonlinear model of the torsion pendulum can be expressed as follows:

$$
\ddot{\theta}+\sum_{j=1}^{n} c_{j}|\dot{\theta}|^{j} \cdot \operatorname{sign}(\dot{\theta})+\sum_{i=1}^{m} k_{i}|\theta|^{i} \cdot \operatorname{sign}(\theta)=0,
$$

where $n$ is the maximum order of the angular velocity and $m$ is the maximum order of the angular displacement.

For convenience, (33) can be rewritten as [11]

$$
\ddot{\theta}+2 h_{0}(t) \dot{\theta}+\omega_{0}^{2}(t) \theta=0 .
$$

If $\theta(t)$ and $h_{0}(t)$ are signals with nonoverlapping spectrum, we can use Hilbert transform for both sides of (34), multiplying each side of the obtained new equation by $j$ and adding it to the corresponding side of (34)

$$
\ddot{\boldsymbol{\beta}}+2 h_{0}(t) \dot{\boldsymbol{\beta}}+\omega_{0}^{2}(t) \boldsymbol{\beta}=0 .
$$

Substitute (29) and (30) into (35) to obtain

$$
\begin{aligned}
& \boldsymbol{\beta}\left[\frac{\ddot{A}}{A}-\omega^{2}+\omega_{0}^{2}+2 h_{0} \frac{\dot{A}}{A}+j\left(2 \frac{\dot{A}}{A} \omega+\dot{\omega}+2 h_{0} \omega\right)\right] \\
& \quad=0 .
\end{aligned}
$$

Solving (36) we can obtain the expressions of instantaneous undamped natural frequency $\omega_{0}(t)$ and instantaneous damping coefficient $h_{0}(t)$ [11]:

$$
\begin{aligned}
& \omega_{0}(t)=\left[\omega^{2}-\frac{\ddot{A}}{A}+\frac{2 \dot{A}^{2}}{A^{2}}+\frac{\dot{A} \dot{\omega}}{A \omega}\right]^{1 / 2} \\
& h_{0}(t)=-\frac{\dot{A}}{A}-\frac{\dot{\omega}}{2 \omega} .
\end{aligned}
$$

The instantaneous characteristics $\omega_{0}(t)$ and $h_{0}(t)$ can be used for the parameter estimation of the nonlinear torsion pendulum system. The relationship between the parameters of (6) and $\omega_{0}(t)$ can be expressed as [21]

$$
\omega_{0}(A)=\left[\sum_{i=1}^{m} \frac{2}{\sqrt{\pi}} \frac{\Gamma(i / 2+1)}{\Gamma((i+1) / 2+1)} k_{i} \cdot A^{i-1}(t)\right]^{1 / 2} .
$$

The parameters $k_{1}$ and $k_{3}$ of (6) can be estimated by the curve fitting of $\omega_{0}(t)$. The moment of inertia can be calculated by $J=K_{1} / k_{1}$, if the value of $K_{1}$ is known.

The relationship between the parameters of $(6)$ and $h_{0}(t)$ can be expressed as [21]

$$
\begin{aligned}
h_{0}(A \omega)= & \sum_{j=0}^{n} \frac{1}{\sqrt{\pi}} \frac{\Gamma(j / 2+1)}{\Gamma((j+1) / 2+1)} c_{j} \\
& \cdot[A(t) \omega(t)]^{j-1} .
\end{aligned}
$$

The parameters $c_{1}$ and $c_{2}$ of (6) can be estimated by the curve fitting of $h_{0}(t)$.

\section{Simulation Analysis}

5.1. Influence of Nonlinear Damping Force. It is known from (21) that the amplitude of the torsion pendulum is related to the damping factor. To analyze the influence of nonlinear damping force, the logarithmic decrement of the angular displacement signals is compared under different damping conditions. By forth-order Runge-Kutta method, (5) is solved to obtain the simulation signals of angular displacement with the parameters in Table 1 . The initial displacement is $0.06 \mathrm{rad}$.

The logarithmic decrement refers to the ratio of the amplitude of adjacent periods, reflecting the degree of amplitude attenuation. The logarithmic decrement of the angular displacement simulation signals is shown in Figure 2. Without the damping force, the logarithmic decrement of $\theta_{1}(t)$ is zero, indicating that the amplitude of the angular displacement does not attenuate. With the linear damping force, the logarithmic decrement of $\theta_{2}(t)$ is 0.014 , indicating that the amplitude of the angular displacement attenuates at a constant rate. With the nonlinear damping force, the logarithmic decrement of $\theta_{3}(t)$ decreases gradually, indicating that the degree of the amplitude attenuation is variable.

5.2. Influence of Nonlinear Restoring Force. It is known from (22) that the period of torsion pendulum is related to the stiffness coefficient of the torsion bar. To analyze the 
TABLE 1: Parameter value of simulation signals under different damping conditions.

\begin{tabular}{cccccc}
\hline & $J$ & $C_{1}$ & $C_{2}$ & $K_{3}$ \\
$\left(\mathrm{~kg} \cdot \mathrm{m}^{2}\right)$ & $\left(\mathrm{kg} \cdot \mathrm{m}^{2} \cdot \mathrm{s}^{-1}\right)$ & $\left(\mathrm{kg} \cdot \mathrm{m}^{2} \cdot \mathrm{rad}^{-1}\right)$ & $\left(\mathrm{N} \cdot \mathrm{m} \cdot \mathrm{rad}^{-1}\right)$ & 5 & 0 \\
\hline$\theta_{1}(t)$ & 1 & 0 & 0 & 5 & 0 \\
$\theta_{2}(t)$ & 1 & 0.01 & 0 & 5 & 0 \\
$\theta_{3}(t)$ & 1 & 0.01 & 0.1 & $\left.5 \cdot \mathrm{rad}^{-3}\right)$ \\
\hline
\end{tabular}

TABLE 2: Parameter value of simulation signals under different stiffness coefficient conditions.

\begin{tabular}{cccccc}
\hline & $J$ & $C_{1}$ & $C_{2}$ & $K_{1}$ \\
$\left(\mathrm{~kg} \cdot \mathrm{m}^{2}\right)$ & $\left(\mathrm{kg} \cdot \mathrm{m}^{2} \cdot \mathrm{s}^{-1}\right)$ & $\left(\mathrm{kg} \cdot \mathrm{m}^{2} \cdot \mathrm{rad}^{-1}\right)$ & 5 & 0 \\
\hline$\theta_{4}(t)$ & 1 & 0.01 & 0 & 5 & 5 \\
$\theta_{5}(t)$ & 1 & 0.01 & 0.1 & 5 & 20 \\
$\theta_{6}(t)$ & 1 & 0.01 & 0.1 & $\left.\mathrm{rad}^{-1}\right)$ & 5 \\
\hline
\end{tabular}

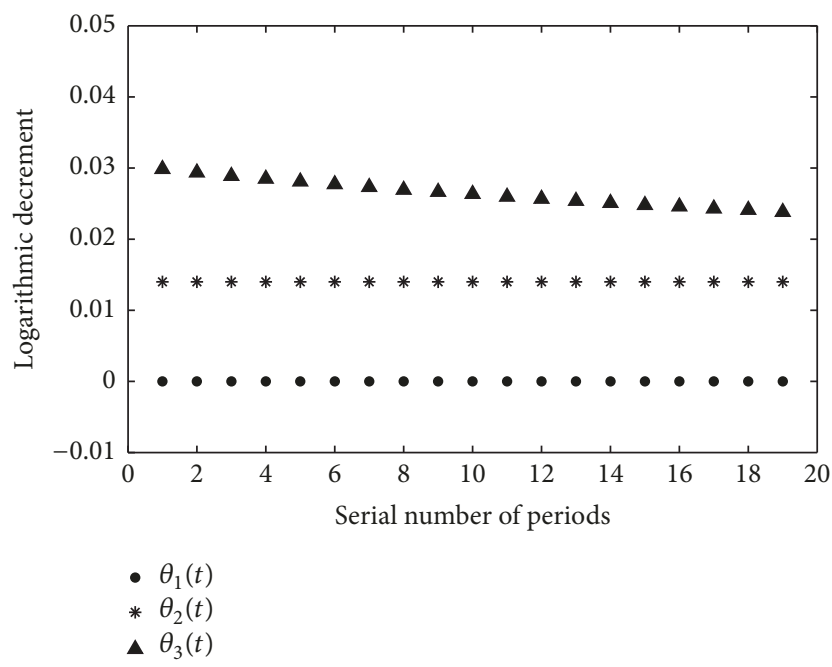

FIGURE 2: Logarithmic decrement of simulation signals.

influence of the nonlinear restoring force, periods of angular displacement signals are compared under different stiffness coefficient conditions. By forth-order Runge-Kutta method, (5) is solved to obtain the simulation signals of angular displacement with parameters in Table 2. The initial displacement is $0.06 \mathrm{rad}$.

The periods of simulation signals under different stiffness coefficient conditions are shown in Figure 3. When $K_{3}=0$, the restoring force of the torsion pendulum is a linear force and the time interval of every period of $\theta_{4}(t)$ is the same. When $K_{3}>0$, the restoring force of the torsion pendulum is a nonlinear force and the time interval of every period of $\theta_{5}(t)$ increases as the amplitude decreases. When $K_{3}<0$, the restoring force of the torsion pendulum is a nonlinear force and the time interval of every period of $\theta_{6}(t)$ decreases as the amplitude decreases. It can be seen that when there is a nonlinear restoring force in the torsion pendulum system, the period of the torsion pendulum will change with the amplitude.

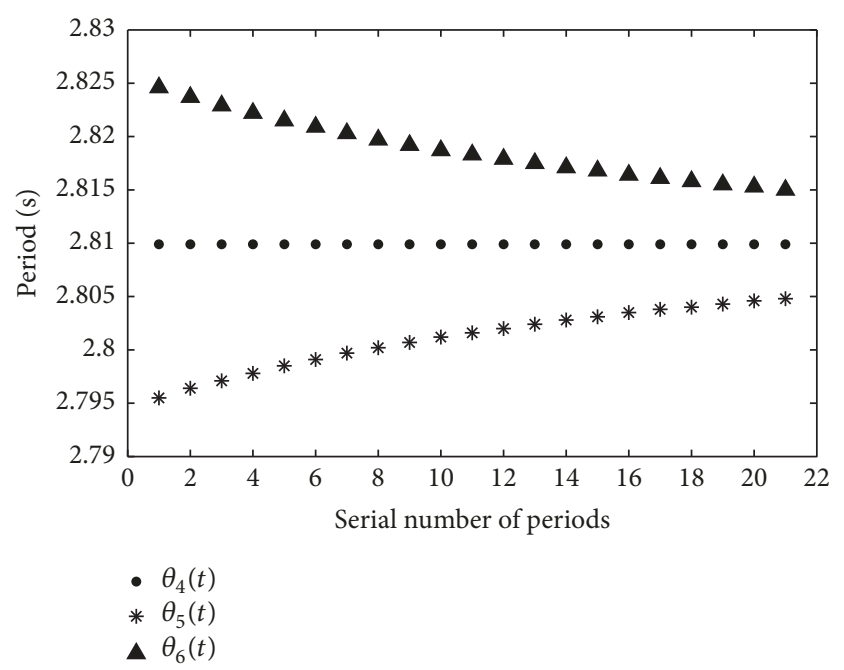

Figure 3: Periods of simulation signal.

5.3. Instantaneous Characteristics. The simulation parameter value of (5) is shown in Table 3. The initial angular displacement is $0.05 \mathrm{rad}$.

The simulation signal of the angular displacement is shown in Figure 4.

According to (25), the analytic signal is formed by the angular displacement signal $\theta_{7}(t)$ and its Hilbert transform. The three-dimensional stereogram of the analytic signal is shown in Figure 5(a). The projection of the analytic signal in each coordinate plane is shown in Figure 5(b). The angular displacement signal $\theta_{7}(t)$ is shown in the plane consisting of real-axis and time-axis. The Hilbert transform of the angular displacement signal is shown in the plane consisting of imaginary-axis and time-axis. The instantaneous envelope obtained according to (28) is shown in Figure 4 (dashed line).

According to (37), the instantaneous undamped natural frequency $\omega_{0}(t)$ is calculated by the instantaneous envelope and instantaneous angular frequency, which is shown in Figure 6. 
TABLE 3: Simulation parameter value of torsion pendulum.

\begin{tabular}{cccccr}
\hline & $\begin{array}{c}J \\
\left(\mathrm{~kg} \cdot \mathrm{m}^{2}\right)\end{array}$ & $\begin{array}{c}C_{1} \\
\left(\mathrm{~kg} \cdot \mathrm{m}^{2} \cdot \mathrm{s}^{-1}\right)\end{array}$ & $\begin{array}{c}\mathrm{C}_{2} \\
\left(\mathrm{~kg} \cdot \mathrm{m}^{2} \cdot \mathrm{rad}^{-1}\right)\end{array}$ & $\begin{array}{c}K_{1} \\
\left(\mathrm{~N} \cdot \mathrm{m} \cdot \mathrm{rad}^{-1}\right)\end{array}$ & $\begin{array}{c}K_{3} \\
\left(\mathrm{~N} \cdot \mathrm{m}^{2} \cdot \mathrm{rad}^{-3}\right)\end{array}$ \\
\hline$\theta_{7}(\mathrm{t})$ & 100 & 1 & 10 & 400 & -600 \\
\hline
\end{tabular}

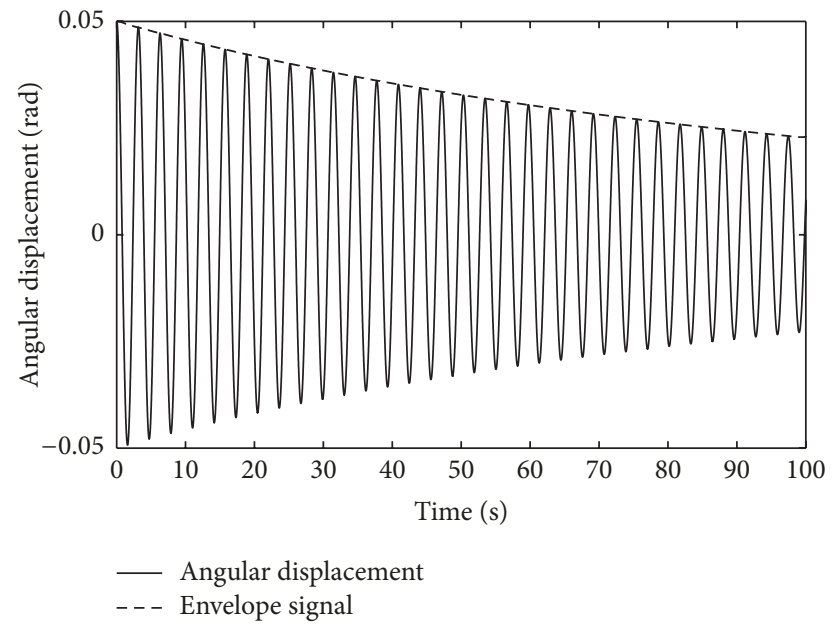

FIGURE 4: Simulation signal of angular displacement.

TABLE 4: Estimation result of nonlinear torsion pendulum system.

\begin{tabular}{lcc}
\hline & Standard value & Estimated value \\
\hline$c_{1}\left(\mathrm{~s}^{-1}\right)$ & 0.0100 & 0.0100 \\
$c_{2}\left(\mathrm{rad}^{-1}\right)$ & 0.1000 & 0.1001 \\
$k_{1}\left(\mathrm{rad}^{-1} \cdot \mathrm{s}^{-2}\right)$ & 4.0000 & 3.9999 \\
$k_{3}\left(\mathrm{rad}^{-3} \cdot \mathrm{s}^{-2}\right)$ & -6.0000 & -6.0043 \\
\hline
\end{tabular}

According to (38), the instantaneous damping coefficient $h_{0}(t)$ is calculated by the instantaneous envelope and instantaneous angular frequency, which is shown in Figure 7.

The instantaneous characteristics $\omega_{0}(t)$ and $h_{0}(t)$ can be used for the parameter estimation of the nonlinear torsion pendulum system. According to (39) and (40), the normalized parameters of the torsion pendulum are estimated by the curve fitting of the curves in Figures 6 and 7. The estimation result is shown in Table 4. If the stiffness coefficient of the torsion bar $K_{1}$ is known, the moment of inertia can be calculated by $J=K_{1} / k_{1}$.

The estimation results under different SNR noise are shown in Table 5.

\section{Experimental Verification}

To verify the correctness of the proposed method, the moment inertia of the experimental sample was measured by the instantaneous characteristics of the torsion pendulum system based on air-hovered turntable. Before the moment of inertia measurement of the experimental sample, the parameters of the torsion pendulum system need to be calibrated, including the stiffness coefficient of the torsion bar and
TABLE 5: Estimation results under different SNR noise.

\begin{tabular}{lcccc}
\hline SNR & $c_{1}\left(\mathrm{~s}^{-1}\right)$ & $c_{2}\left(\mathrm{rad}^{-1}\right)$ & $k_{1}\left(\mathrm{rad}^{-1} \cdot \mathrm{s}^{-2}\right)$ & $k_{3}\left(\mathrm{rad}^{-3} \cdot \mathrm{s}^{-2}\right)$ \\
\hline $60 \mathrm{~dB}$ & 0.0100 & 0.1000 & 4.0001 & -6.0173 \\
$50 \mathrm{~dB}$ & 0.0100 & 0.1002 & 3.9994 & -5.9731 \\
$40 \mathrm{~dB}$ & 0.0098 & 0.1032 & 3.9979 & -5.9592 \\
\hline
\end{tabular}

the moment of inertia of the turntable under the no-load condition. The angular displacement signal of the turntable is measured by an incremental encoder using a grating disk. The sampling frequency is $300 \mathrm{~Hz}$.

6.1. Parameter Calibration of Torsion Pendulum. As shown in Figure 8, the parameters of the torsion pendulum system are calibrated by the standard weight with known moment of inertia. The weight is made of iron cylinder with uniform density. Because of the uniform density, regular shape and high machining accuracy, the weight moment of inertia can be accurately calculated according to the external dimensions and quality of the weight. By the theoretical calculation, the total moment of inertia of weight $A$ and weight $B$ about the rotation axis is $57.3002 \mathrm{~kg} \cdot \mathrm{m}^{2}$.

The normalized parameters of the torsion pendulum model under the no-load condition can be estimated by the instantaneous characteristics of the torsion pendulum. If the moment of inertia of the torsion pendulum under the no-load condition is $J_{0}$, then

$$
\begin{aligned}
& k_{11}=\frac{K_{1}}{J_{0}} \\
& k_{31}=\frac{K_{3}}{J_{0}},
\end{aligned}
$$

where $k_{11}$ and $k_{31}$ are the normalized stiffness coefficients of the torsion bar under the no-load condition.

As shown in Figure 8, two identical standard weights are symmetrically placed on either side of the shaft. The normalized parameters of the torsion pendulum model under the standard weight condition can be estimated by the instantaneous characteristics of the torsion pendulum. If the moment of inertia of weight $A$ and weight $B$ is expressed as $J_{A}$ and $J_{B}$, respectively, then

$$
\begin{aligned}
& k_{12}=\frac{K_{1}}{J_{0}+J_{A}+J_{B}} \\
& k_{32}=\frac{K_{3}}{J_{0}+J_{A}+J_{B}},
\end{aligned}
$$

where $k_{12}$ and $k_{32}$ are the normalized stiffness coefficients of the torsion bar under the weight condition. 


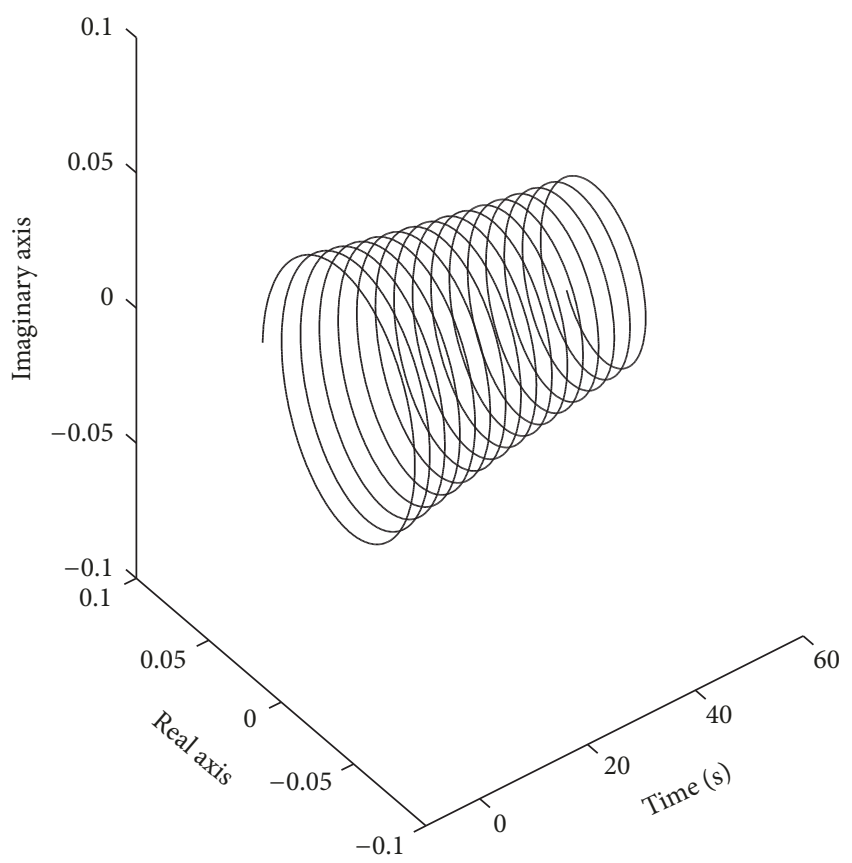

(a) Stereogram of analytic signal

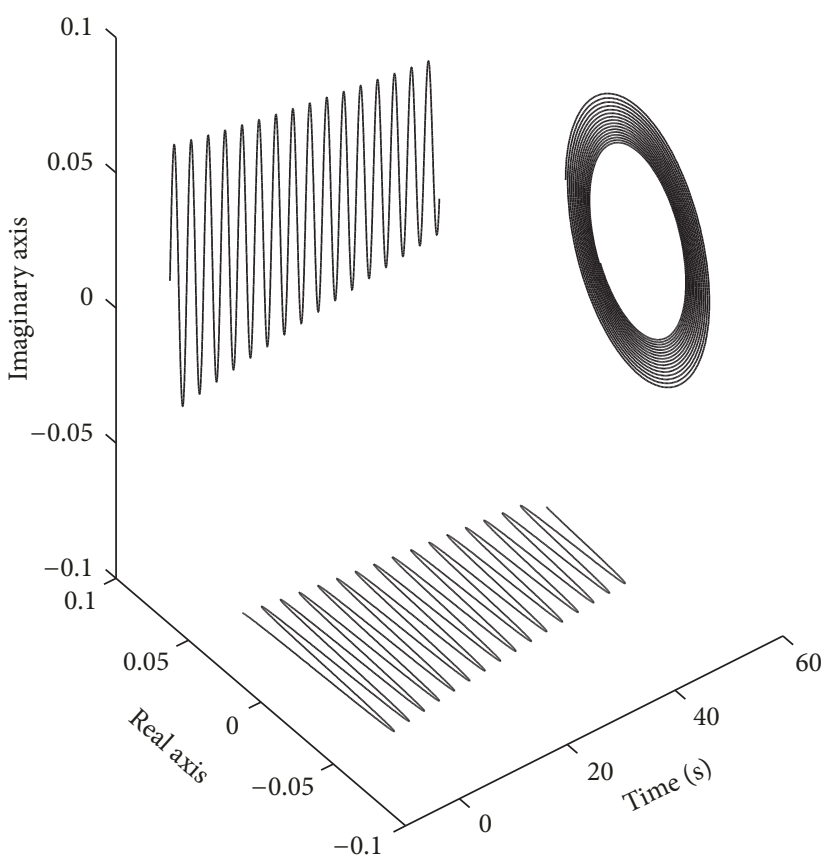

(b) Projection graph of analytic signal

FIGURE 5: Stereogram and projection graph of analytic signal.

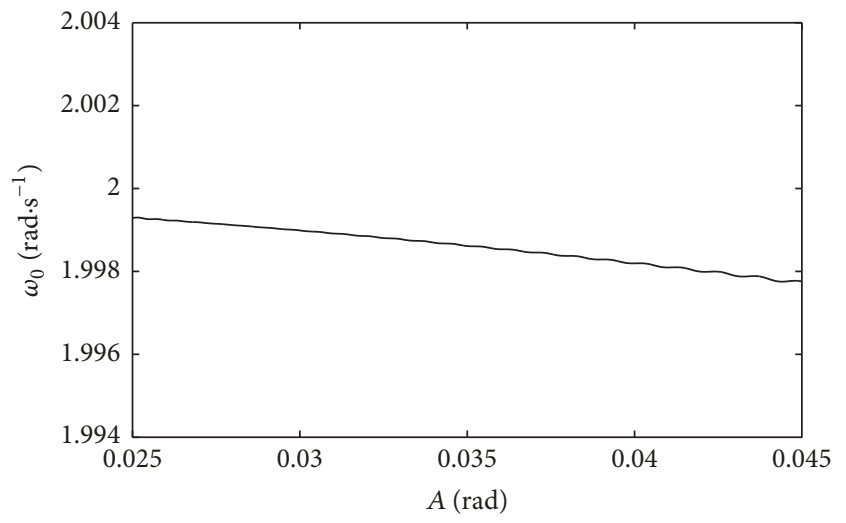

FigURE 6: Instantaneous undamped natural frequency.

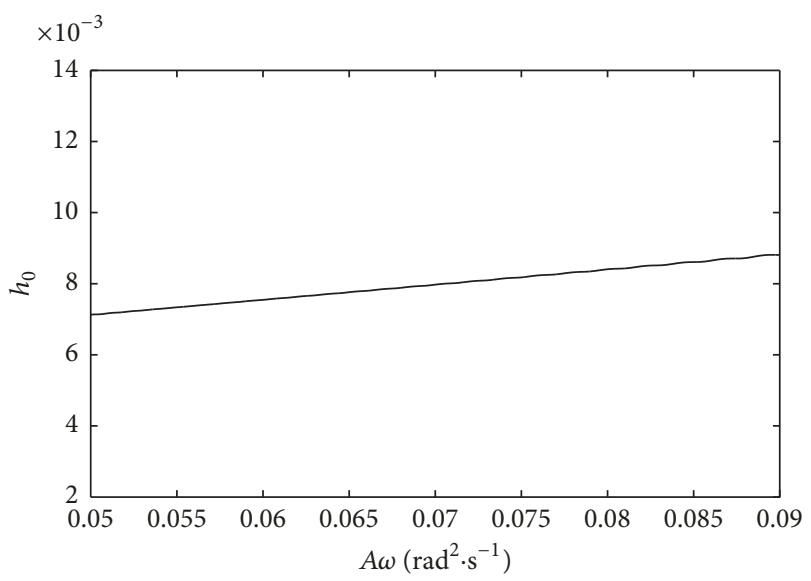

FIGURE 7: Instantaneous damping coefficient.

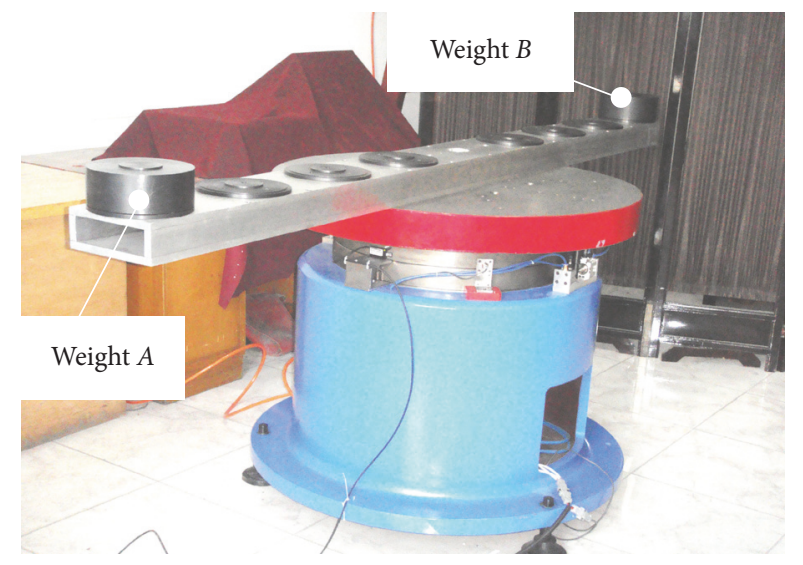

FIGURE 8: Calibration system of the torsion pendulum.

According to (41) and (42), the parameters of the torsion pendulum system can be expressed as

$$
\begin{aligned}
& K_{1}=\frac{k_{11} \cdot k_{12} \cdot\left(J_{A}+J_{B}\right)}{k_{11}-k_{12}} \\
& K_{3}=\frac{k_{31} \cdot k_{12} \cdot\left(J_{A}+J_{B}\right)}{k_{11}-k_{12}} \\
& J_{0}=\frac{k_{12} \cdot\left(J_{A}+J_{B}\right)}{k_{11}-k_{12}} .
\end{aligned}
$$

The calibration results of the torsion pendulum system are shown in Table 6. 
TABLE 6: Calibration results of torsion pendulum.

\begin{tabular}{lccc}
\hline & $K_{1} /\left(\mathrm{N} \cdot \mathrm{m} \cdot \mathrm{rad}^{-1}\right)$ & $K_{3} /\left(\mathrm{N} \cdot \mathrm{m} \cdot \mathrm{rad}^{-3}\right)$ & $J_{0} /\left(\mathrm{kg} \cdot \mathrm{m}^{2}\right)$ \\
\hline$(1)$ & 467.0433 & -2212.7707 & 94.6332 \\
$(2)$ & 467.1702 & -2211.7868 & 94.6616 \\
$(3)$ & 467.2111 & -2200.9289 & 94.6730 \\
\hline
\end{tabular}

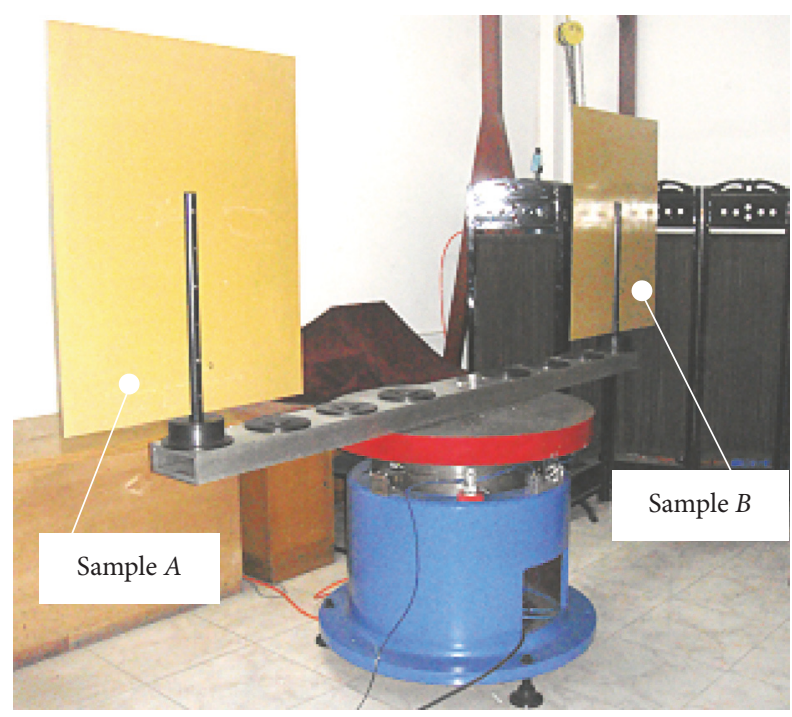

FIGURE 9: Moment of inertia measurement system.

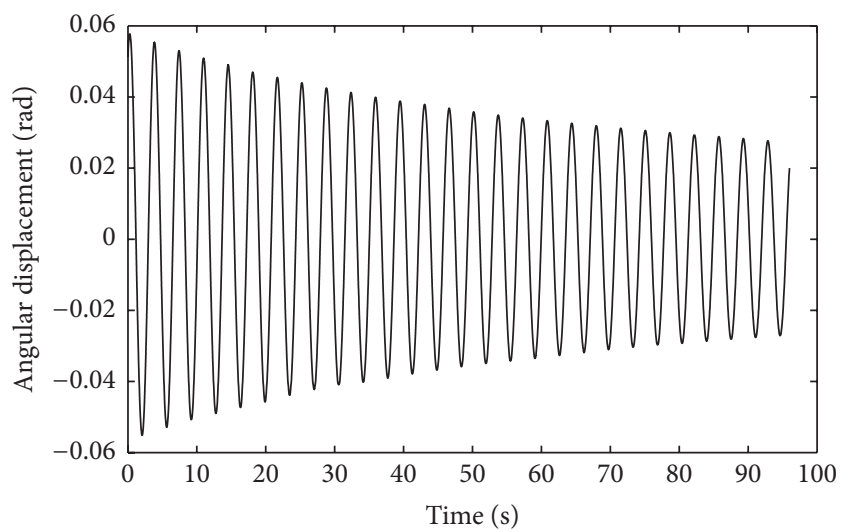

FIGURE 10: Experimental angular signal of turntable.

6.2. Experimental Result. As shown in Figure 9, two identical samples with large outline size are symmetrically placed on the both sides of the rotation axis, which can enhance the air damping force. By the theoretical calculation, the total moment of inertia of the samples about the rotation axis is $53.9056 \mathrm{~kg} \cdot \mathrm{m}^{2}$. By the calibration of the standard weight, the stiffness coefficient of the torsion bar is $K_{1}=$ $467.1702 \mathrm{~N} \cdot \mathrm{m} \cdot \mathrm{rad}^{-1}, K_{3}=-2211.7868 \mathrm{~N} \cdot \mathrm{m} \cdot \mathrm{rad}^{-3}$, and the moment of inertia of the turntable is $94.6616 \mathrm{~kg} \cdot \mathrm{m}^{2}$.

The angular displacement signal of the turntable is shown in Figure 10. To analyze the influence of nonlinear damping force and nonlinear restoring force, it is necessary to calculate the period and logarithmic decrement of the angular displacement signal.

The logarithmic decrement of the experimental signal is shown in Figure 11. It is known from Figure 11 that the

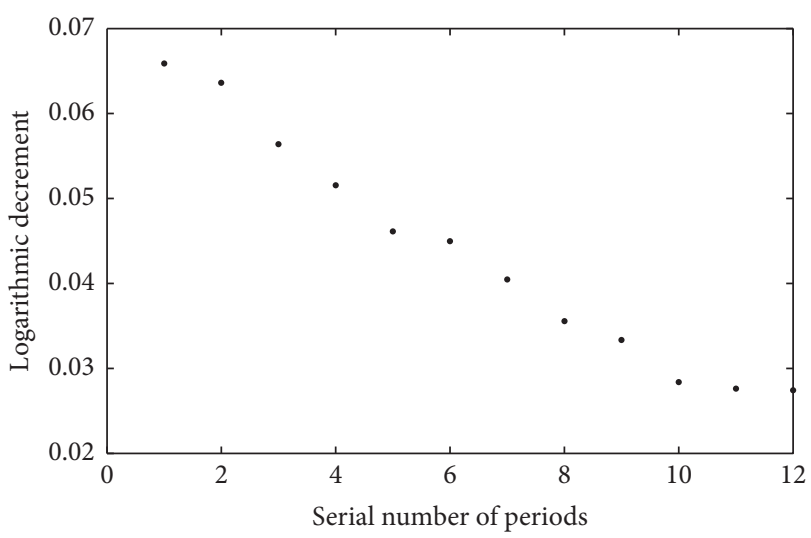

FIGURE 11: Logarithmic decrement of experimental signal.

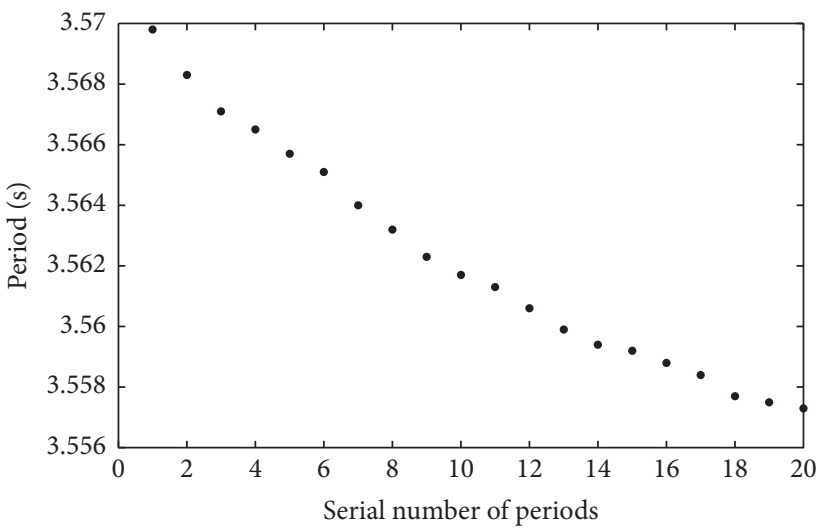

Figure 12: Periods of experimental signal.

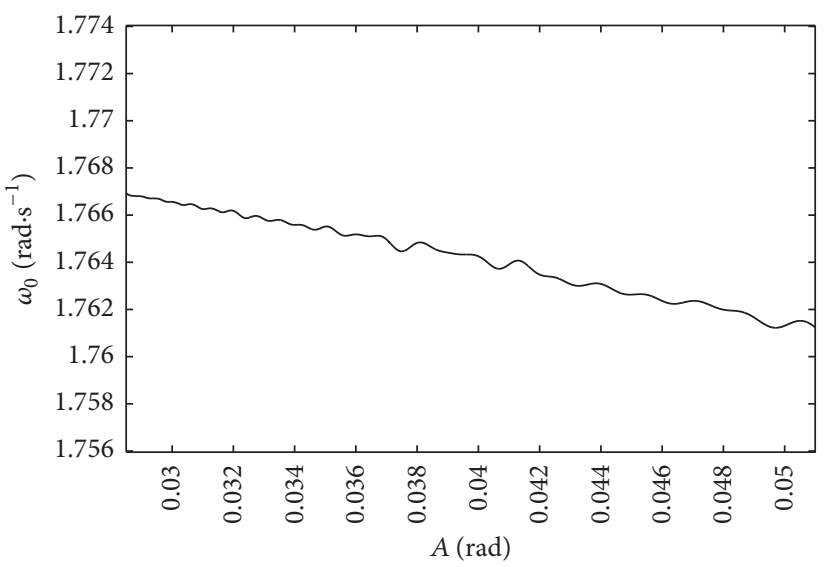

FIGURE 13: Instantaneous undamped natural frequency.

logarithmic decrement of the experimental signal decreases gradually, which is consistent with the simulation result of the nonlinear model of the torsion pendulum.

The time intervals of the experimental signal are shown in Figure 12. It is known from Figure 12 that the periods of the experimental signal decrease gradually, which is consistent with the simulation result of the nonlinear model of the torsion pendulum.

The instantaneous undamped natural frequency $\omega_{0}(t)$ and instantaneous damping coefficient $h_{0}(t)$ of the experimental system are shown in Figures 13 and 14. 
TABLE 7: Relative error of moment of inertia.

\begin{tabular}{lcc}
\hline & Estimated value & Relative error \\
\hline$(1)$ & $54.2722 \mathrm{~kg} \cdot \mathrm{m}^{2}$ & $0.68 \%$ \\
$(2)$ & $54.2236 \mathrm{~kg} \cdot \mathrm{m}^{2}$ & $0.59 \%$ \\
$(3)$ & $54.2021 \mathrm{~kg} \cdot \mathrm{m}^{2}$ & $0.55 \%$ \\
$(4)$ & $54.2398 \mathrm{~kg} \cdot \mathrm{m}^{2}$ & $0.62 \%$ \\
$(5)$ & $54.2129 \mathrm{~kg} \cdot \mathrm{m}^{2}$ & $0.57 \%$ \\
\hline
\end{tabular}

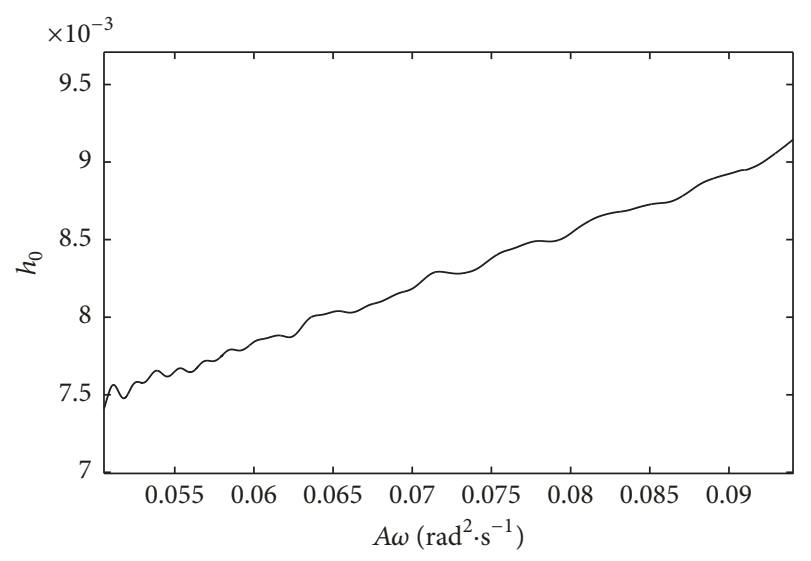

FIGURE 14: Instantaneous damping coefficient.

The parameter estimation method based on the instantaneous characteristics can be validated by the measurement of the moment of inertia of the samples. According to (39), the measured value of $k_{1}$ is 3.1368 by the curve fitting of the instantaneous undamped natural frequency in Figure 13. As the stiffness coefficient $K_{1}$ is known, the measured value of the total moment of inertia of samples and turntable is $148.9321 \mathrm{~kg} \cdot \mathrm{m}^{2}$. As the moment of inertia of the turntable is known, the measured value of the total moment of inertia of samples is $54.2705 \mathrm{~kg} \cdot \mathrm{m}^{2}$.

The moment of inertia of the samples is measured by 5 times. As shown in the result in Table 7, the relative error of the moment of inertia of the samples is within $0.7 \%$, which verifies the effectiveness of the parameter estimation method based on the instantaneous characteristics.

The main factors affecting the measurement results include the calibration of the torsion pendulum, the theoretical value of the moment of inertia of the samples, the environmental noise, and the sampling accuracy. The method of improving measurement accuracy will be carried out in future research.

\section{Conclusion}

Considering the influence of nonlinear damping force and nonlinear restoring force, the nonlinear torsion pendulum model is established. The analytic solution of the nonlinear model is solved by the average method. The simulation results show that the logarithmic decrement of the torsion pendulum varies by the nonlinear damping force and the period of the torsion pendulum varies by the nonlinear restoring force. The instantaneous characteristics can be used for the parameter estimation of the nonlinear torsion pendulum system. The torsion pendulum system based on the air-hovered turntable verifies the nonlinear characteristics of the torsion pendulum.

\section{Conflicts of Interest}

All authors declare that there are no conflicts of interest regarding the publication of this paper.

\section{Acknowledgments}

This research is supported by the Research Project of Tianjin Education Committee (no. 2017KJ253), the National Nature Science Foundation of China (no. 61502340), and the Science Foundation of Tianjin Key Laboratory for Control Theory and Applications in Complicated Systems (no. TJKL-CTACS201707).

\section{References}

[1] C. Schedlinski and M. Link, "Survey of current inertia parameter identification methods," Mechanical Systems and Signal Processing, vol. 15, no. 1, pp. 189-211, 2001.

[2] J. Wang, R. Li, and X. Peng, "Survey of nonlinear vibration of gear transmission systems," Applied Mechanics Reviews, vol. 56, no. 3, pp. 309-329, 2003.

[3] J. L. Felix, J. M. Balthazar, and R. M. Brasil, "A short note on transverse vibrations of a shaft carrying two (or one) disk excited by a nonideal motor," Journal of Computational and Nonlinear Dynamics, vol. 4, no. 1, pp. 53-63, 2009.

[4] L. Klaus, "Comparison of two experiments based on a physical and a torsion pendulum to determine the mass moment of inertia including measurement uncertainties," Measurement Science Review, vol. 17, no. 1, pp. 9-18, 2017.

[5] M. R. Jardin and E. R. Mueller, "Optimized measurements of unmanned-air-vehicle mass moment of inertia with a bifilar pendulum," Journal of Aircraft, vol. 46, no. 3, pp. 763-775, 2009.

[6] L. Cveticanin, "Resonant vibrations of nonlinear rotors," Mechanism and Machine Theory, vol. 30, no. 4, pp. 581-588, 1995.

[7] A. H. Nayfeh, Introduction to Perturbation Techniques, John Wiley \& Sons, New York, NY, USA, 1981.

[8] H. Wang and H. Hu, "Remarks on the perturbation methods in solving the second-order delay differential equations," Nonlinear Dynamics, vol. 33, no. 4, pp. 379-398, 2003.

[9] N. Bogoliubov and A. Mitropolsky, Asymptotic Methods in the Theory of Nonlinear Oscillations, Gordon and Breach, 1961.

[10] N. Parandin, "Numerical solution of fuzzy differential equations of nth-order by Runge-Kutta method," Neural Computing and Applications, vol. 21, no. 1, pp. 347-355, 2012.

[11] M. Feldman, "Non-linear system vibration analysis using Hilbert transform-I. Free vibration analysis method 'FREEVIB'”, Mechanical Systems and Signal Processing, vol. 8, no. 2, pp. 119127, 1994.

[12] M. Feldman, "Non-linear system vibration analysis using Hilbert transform-II. Forced vibration analysis method 'Forcevib"' Mechanical Systems and Signal Processing, vol. 8, no. 3, pp. 309-318, 1994.

[13] Y. Avargel and I. Cohen, "Adaptive nonlinear system identification in the short-time Fourier transform domain," IEEE 
Transactions on Signal Processing, vol. 57, no. 10, pp. 3891-3904, 2009.

[14] Y. Avargel and I. Cohen, "Modeling and identification of nonlinear systems in the short-time Fourier transform domain," IEEE Transactions on Signal Processing, vol. 58, no. 1, pp. 291304, 2010.

[15] B. Ergen, Y. Tatar, and H. O. Gulcur, "Time-frequency analysis of phonocardiogram signals using wavelet transform: A comparative study," Computer Methods in Biomechanics and Biomedical Engineering, vol. 15, no. 4, pp. 371-381, 2012.

[16] A. Briassouli, D. Matsiki, and I. Kompatsiaris, "Continuous wavelet transform for time-varying motion extraction," IET Image Processing, vol. 4, no. 4, pp. 271-282, 2010.

[17] L. Xiang and A. Hu, "Comparison of methods for different timefrequency analysis of vibration signal," Journal of Software, vol. 7, no. 1, pp. 68-74, 2012.

[18] S. Qian and D. Chen, "Decomposition of the Wigner-Ville Distribution and Time-Frequency Distribution Series," IEEE Transactions on Signal Processing, vol. 42, no. 10, pp. 2836-2842, 1994.

[19] N. E. Huang, M. C. Wu, S. R. Long et al., "A confidence limit for the empirical mode decomposition and Hilbert spectral analysis," Proceedings of Royal Society of London, Series A, vol. 459, no. 2037, pp. 2317-2345, 2003.

[20] Y. Liu and L. Chen, Nonlinear Vibration, Higher Education Press, Beijing, China, 2001.

[21] L. Wang, J. Zhang, C. Wang, and S. Hu, "Time-frequency analysis of nonlinear systems: The skeleton linear model and the skeleton curves," Journal of Vibration and Acoustics, vol. 125, no. 2, pp. 170-177, 2003. 


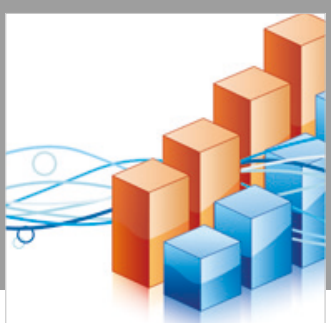

Advances in

Operations Research

\section{-n-m}
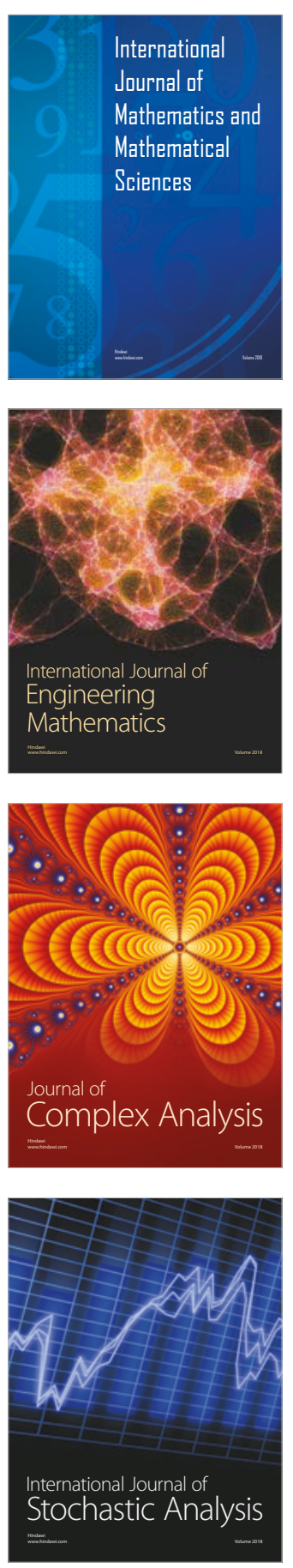
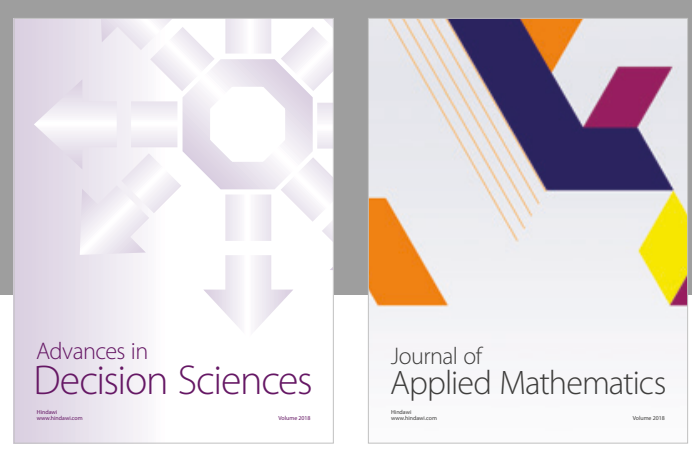

Journal of

Applied Mathematics
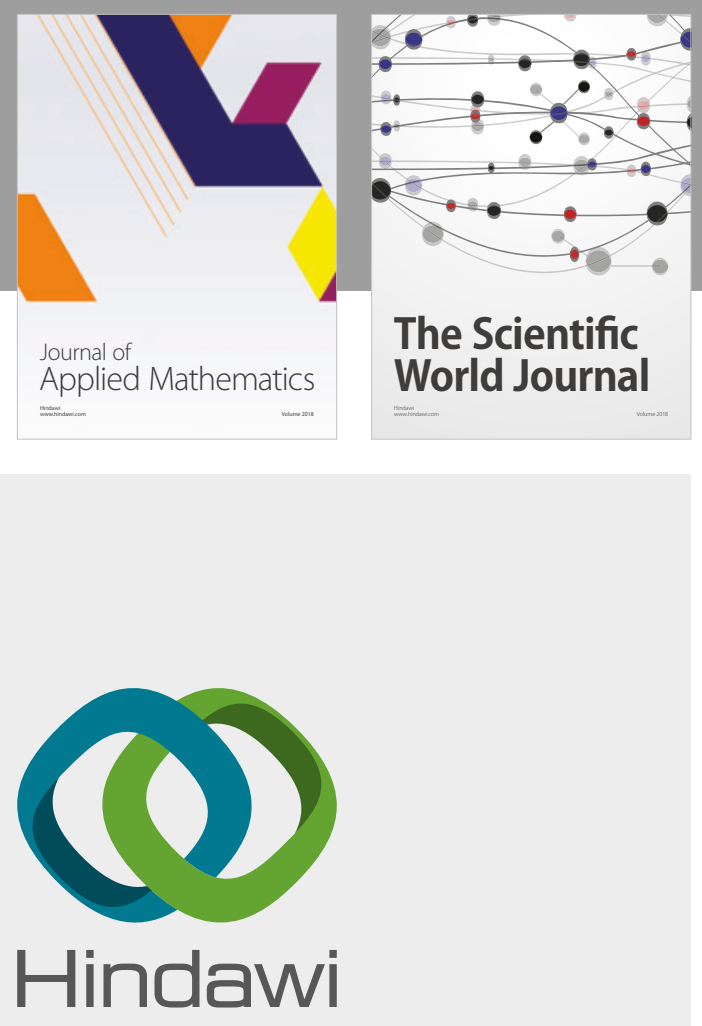

Submit your manuscripts at

www.hindawi.com

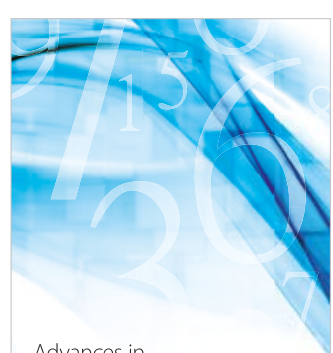

Advances in
Numerical Analysis
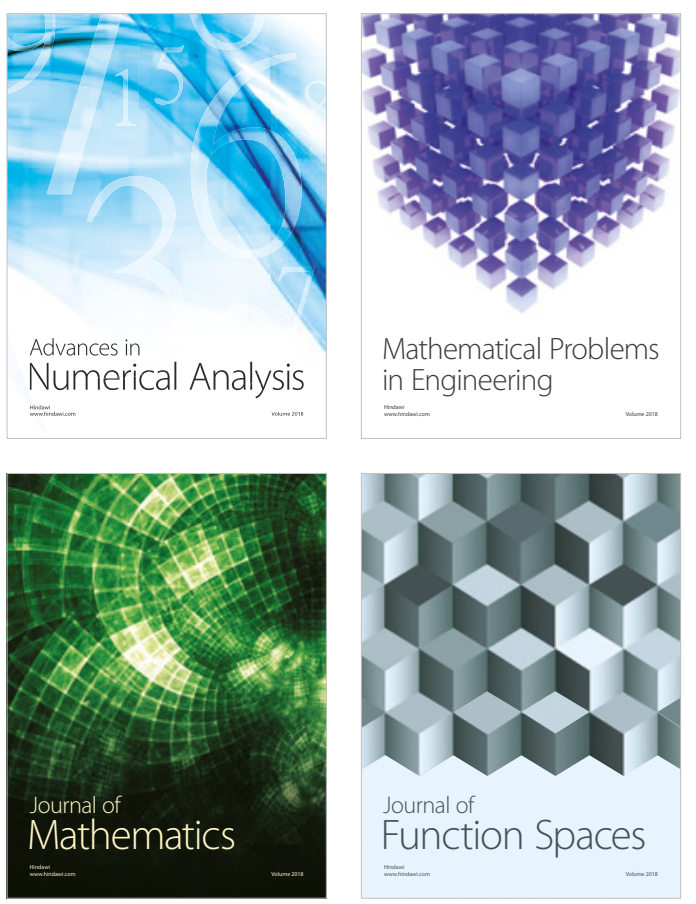

Mathematical Problems in Engineering

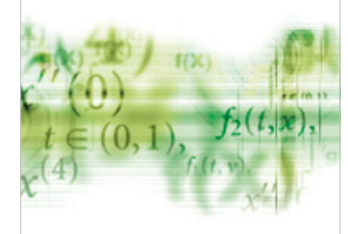

International Journal of

Differential Equations

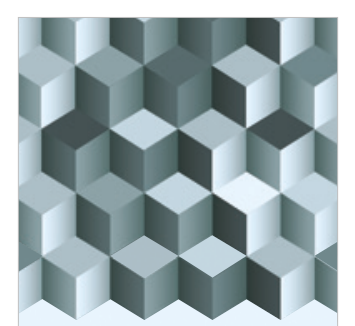

Journal of

Function Spaces

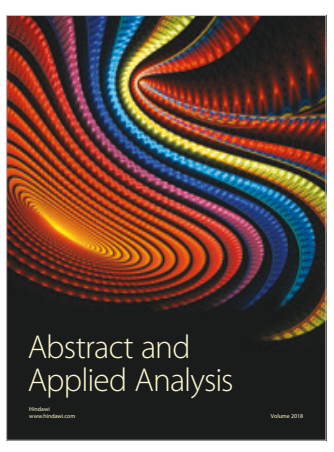

The Scientific

World Journal

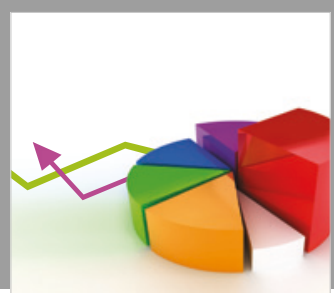

Journal of

Probability and Statistics
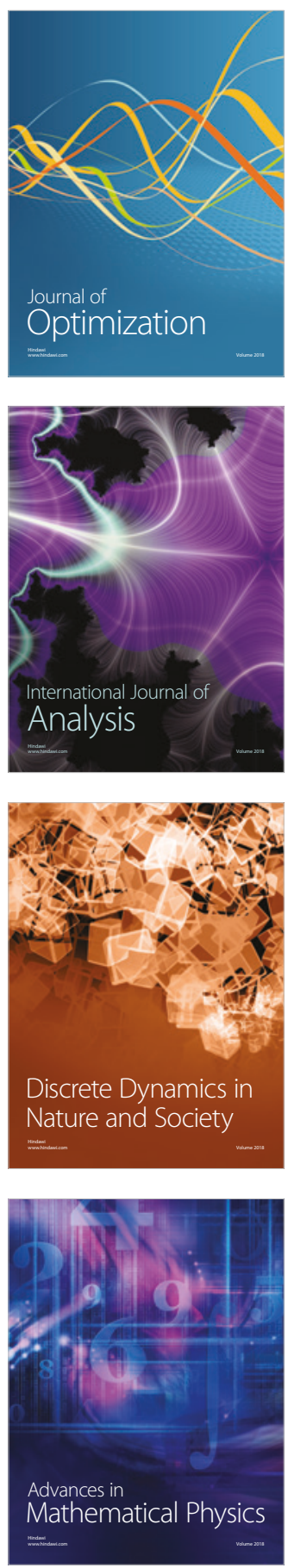